Supporting Information

\title{
Increasing Collisional Activation of Protein Complexes Using Smaller Aperture Source Sampling Cones on a Synapt Q-IM-TOF Instrument with a Stepwave Source
}

Jesse W. Wilson ${ }^{\mathrm{a}}$, Micah T. Donora, Samantha O. Shepherd ${ }^{\mathrm{a}}$, James S. Prella,b*

${ }^{a}$ Department of Chemistry and Biochemistry, University of Oregon, 1253 University of Oregon Eugene, OR, USA, 97403-1253

${ }^{b}$ Materials Science Institute, University of Oregon, 1252 University of Oregon, OR, USA, 97403-1252

Corresponding author: James S. Prell

address: 1253 University of Oregon, Eugene, OR, USA, 97403-1253

phone: 541-346-2597

email: jprell@uoregon.edu

Submitted to Journal of the American Society for Mass Spectrometry

April 6, 2020

Revised July 2, 2020 
Table of Contents.

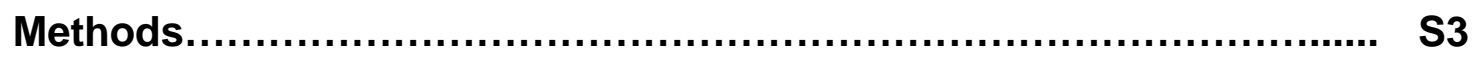

Simulations of in-source activation..................................... S6

Supplemental figures and tables.......................................... S10 


\section{Methods.}

GroEL mass spectral acquisition and data analysis. For each trial day (1-3), previously prepared frozen aliquots of GroEL were buffer exchanged using a Bio-Rad Micro Bio-Spin 6 Columns (Ca.\#732-6221) equilibrated with 200 mM ammonium acetate, $\mathrm{pH}$ 7.5. Borosilicate glass capillary emitters $(1.0 \mathrm{~mm}$ o.d./0.78 mm i.d., Sutter Instruments) were pulled using a Flaming/Brown micropipette puller (Model P-97, Sutter Instruments) to a tip size of $\sim 2 \mu \mathrm{m}$ for nanoelectrospray ionization (nESI). About $3 \mu \mathrm{L}$ of sample was loaded into the pulled capillaries. Spray was initiated with a Pt wire inserted into the sample solution at a capillary voltage of $\sim 1.0 \mathrm{kV}$. The capillary voltage was then reduced to $\sim 0.65 \mathrm{kV}$ for data collection.

Before the start of every trial the nESI capillary was positioned such that the maximum GroEL signal was achieved for each cone. Typically, the highest signal was reached with the capillary positioned $\sim 2 \mathrm{~mm}$ from the sampling cone holder (orthogonal to the cone). No significant effect on excess mass or desalting was observed when adjusting the capillary position across the small range of distances used. GroEL mass spectra were recorded for each source sampling cone (in mixed order across trial days) with the same instrumental parameters (see below), while the sampling cone voltage was raised from 10-180 V in $10 \mathrm{~V}$ increments. At each sampling cone voltage step, a 1minute acquisition was acquired and summed so that GroEL signal abundances could be compared for each cone and trial day. When exchanging each sample cone for the next trial, the new cone was allowed to equilibrate temperature with the rest of the source block for $\sim 5$ minutes and a new nESI capillary with GroEL sample was used. 
Spray of these samples is consistent across the range of time needed to collect a trial run (under $60 \mathrm{~min}$.). The standard deviations in excess mass are below $1 \mathrm{kDa}$ at the high end, which is observed at lower source sampling cone voltages. At high cone voltages, the standard deviations are smaller (under $100 \mathrm{Da}$ ), at the end of a trial run. Thus the spray conditions are consistent through the experiment and random order of the cone voltages is not necessary.

IM-MS spectra for each trial day were then analyzed with Gaussian multi-peak fitting in Igor Pro to determine the average mass, peak width (fwhm), and abundances of each charge state. The entire drift time profile for each charge state was extracted and the average weighted drift time was calculated for a given charge state so that compaction and/or unfolding could be observed across source sampling cones and sampling cone potentials.

$\alpha H L$ sample preparation. Lyophilized monomeric alpha hemolysin ( $\alpha \mathrm{HL})$ from $S$. aureus was purchased from Millipore-Sigma (St. Louis, MO, USA). To produce aHL pores, lyophilized monomers were resuspended in water to a concentration of 0.5 $\mathrm{mg} / \mathrm{mL} .150 \mu \mathrm{L}$ of this solution was then mixed with $4 \mu \mathrm{L}$ of $100 \mathrm{mM} \mathrm{n}$ tetradecylphosphocholine (FOS-14) stock solution to reach a final detergent concentration of $2 \mathrm{mM}$ Fos-14 and enough ammonium acetate solution to reach a total sample volume of $200 \mu \mathrm{L}$. This mixture of $\alpha \mathrm{HL}$ monomer and detergent was then concentrated in a $3 \mathrm{kDa}$ cutoff centrifugal concentrator to an approximate volume of 35$40 \mu \mathrm{L}$. After concentration, the sample was aliquoted to $10 \mu \mathrm{L}$ aliquots. Before use, each $10 \mu \mathrm{L}$ aliquot was diluted with $20 \mu \mathrm{L}$ of 2 mM FOS-14 in $200 \mathrm{mM}$ ammonium acetate, $\mathrm{pH}$ 7.5. Then the sample was buffer exchanged using a centrifugal desalting 
column equilibrated with $0.25 \mathrm{mM}$ FOS-14 (2x critical micelle concentration) in $200 \mathrm{mM}$ ammonium acetate $\mathrm{pH}$ 7.5. MS analysis was performed immediately after buffer exchanging samples using TOF mode without IM.

\section{Synapt G2-Si instrument parameters.}

Source Temperature: $100^{\circ} \mathrm{C}$

Source sampling cone voltage: $10-180 \mathrm{~V}$ with $10 \mathrm{~V}$ steps

Quadrupole profile was set to: $3000,6000,10000$

Trap voltage: $10 \mathrm{~V}$ for GroEL experiments, $50 \mathrm{~V}$ for $\alpha \mathrm{HL}$ experiments

Transfer voltage: $5 \mathrm{~V}$ for all experiments

Trap Argon gas flow rate: $10 \mathrm{~mL} / \mathrm{min}$

Helium cell flow rate: $50 \mathrm{~mL} / \mathrm{min}$

IMS Nitrogen flow rate: $100 \mathrm{~mL} / \mathrm{min}$

Traveling Wave settings for GroEL experiments:

Trap wave velocity: $300 \mathrm{~m} / \mathrm{s}$

Trap wave height: $1.0 \mathrm{~V}$

IMS wave velocity: $400 \mathrm{~m} / \mathrm{s}$

IMS wave height: $18 \mathrm{~V}$

Transfer wave velocity: $100 \mathrm{~m} / \mathrm{s}$

Transfer wave height: $2.0 \mathrm{~V}$

Traveling Wave settings for $\mathrm{\alpha HL}$ experiments performed in ToF mode without IM:

Trap wave velocity: $300 \mathrm{~m} / \mathrm{s}$

Trap wave height: $1.0 \mathrm{~V}$

Transfer wave velocity: $100 \mathrm{~m} / \mathrm{s}$ 
Transfer wave height: $2.0 \mathrm{~V}$

*The Synapt instrument was modified according to Waters instructions to extend the source sampling cone voltage maximum from $150 \mathrm{~V}$ to $200 \mathrm{~V}$ by changing MassLynx parameters, for more details one should contact Waters.

\section{Simulations of in-source ion activation.}

Simulations were conducted according to the method described in Donor et al. ${ }^{11}$, with limited modifications to model the conditions in the source region. The collision physics remained the same as in our previous work. Briefly, collisions between the ion and gas were assumed to be inelastic, and $90 \%$ of the available center-of-mass-frame kinetic energy converted to internal energy of the ion. The collision geometry and gas velocity were randomly sampled from uniform and Boltzmann distributions, respectively. Mean free path is determined from the ion CCS, and initial velocity from the ion mass and charge and acceleration voltage. Ion velocity and internal energy were updated following each collision. In addition to increasing (heating), the ion internal energy can also decrease (cooling). This is accomplished via equipartition of the total internal energy among the modes of a collision complex between the ion and gas. Due to the different conditions in the Stepwave and Source ion guide compared to those modeled in the original simulations, changes to the ion acceleration, pressure profile, and time step were implemented in the simulations performed in this work.

Ion Acceleration. The ions are accelerated across the $\sim 1 \mathrm{~mm}$ gap between the exit of the Stepwave and the differential aperture, an area with gas pressure in the mbar range (estimated using the Backing pressure readback, $\sim 3.0$ mbar for the large SC). Thus, rather than assume that the ions reach a kinetic energy equal to the charge 
multiplied by the acceleration potential, the acceleration was modeled explicitly in these simulations. The ions were assumed to have an initial velocity equal to their RMS thermal velocity at $298 \mathrm{~K}(\sim 10 \mathrm{~m} / \mathrm{s})$, and the change in kinetic energy for a time step was taken as the charge multiplied by the change in potential over the distance traveled during that time step. Once the ions travel $1 \mathrm{~mm}$, they enter the Source ion guide $(\sim 9$ $\mathrm{cm}$ in length) and experience only the traveling-wave potential (wave velocity of $300 \mathrm{~m} / \mathrm{s}$ and wave height of $1 \mathrm{~V}$ ) for the rest of the simulation.

Pressure Profile. While our previous simulations of heating modeled the Trap region of a Synapt G2-Si, which can be treated as a region of constant pressure, the current simulations model heating in a region of changing pressure. The Stepwave is held at a higher pressure than the Source ion guide, and the pressure differential between the two regions was assumed to follow an exponential profile. A new parameter, the characteristic decay length, was introduced and set equal to the diameter of the differential aperture between the Stepwave and Source ion guide regions ( 2.5 mm). Shorter (Fig. S1a, $1.25 \mathrm{~mm}$ ) and longer (Fig. S1b, $5 \mathrm{~mm}$ ) exponential decay lengths of the pressure do not change the trends in the slopes of the ion heating plots, but the slopes do get closer together for the $1.25 \mathrm{~mm}$ decay length and further apart for the $5.0 \mathrm{~mm}$ decay length due to decreased/increased cooling, respectively.

Time Step. In the original code, the time step (ts) was calculated based on the ion's initial (i.e., maximum) velocity as ts $=(1 / 20)$ (mean free path $) /($ max velocity). That is, the time step is the shortest amount of time it would take the ion to move $1 / 20$ th of the mean free path. This same method was used here to determine the time step while 
the ions are being quickly accelerated. In order to reduce simulation time, time steps after the initial acceleration were determined at each step using the current velocity.

Acceleration voltages of $25,50,75,100,125,150,175$, and $200 \mathrm{~V}$ were simulated for each of the three pressures. Although GroEL was used for the experimental measurements, there are several reasons why it may not be accurately modeled by our simulations. The pressures at the beginning of the simulations (representing the latter half of the Stepwave) are high enough that simultaneous collisions may occur for GroEL, which our model does not account for. In addition, our model for cooling assumes equipartition of energy between the modes of a collision complex involving the ion and gas. However, GroEL is large enough that this assumption may break down, and energy may be more localized on the timescale of the collisions. Thus, modeling GroEL in these conditions would require additional details of the collision process that are not well understood. Instead, BSA, a smaller ( $67 \mathrm{kDa})$ protein was used, to avoid the issues described above. A charge state of $15+$ was used, corresponding to the most abundant charge state for BSA electrosprayed from nativelike solution conditions.

Due to the model for heating used in the simulations, even at low velocities a small amount of internal energy will be transferred to the ion in the majority of collisions. In addition, below a certain threshold cooling due to equipartition of energy is relatively slow. Thus, at low acceleration voltages (low velocity), the number of collisions is the predominant factor controlling the overall energy deposition, and thus higher pressures lead to a greater change in internal energy. At higher voltages (velocities), the amount of energy transferred exceeds the threshold at which cooling begins to play a significant 
role, and the lower pressure simulations yield greater changes in internal energy due to fewer collisions (opportunities for cooling). The crossover point occurs when these competing factors approximately balance each other. However, in these simulations, the slope has been better validated than the intercept, and we are cautious about overinterpreting the simulated intercepts, which may be less trustworthy than the slopes.

From these simulations (Figure S7), little difference in effective ion internal temperature is predicted as a function of pressure at low cone potentials. However, the slopes of the ion heating trends increase as the pressure is reduced with the medium (slope $1.5 x$ large) and small (slope $2.25 x$ large) cones. This means as the cone potential is raised at lower pressures, a higher percentage of collisions are net heating leading to a more efficient conversion of kinetic to internal energy, consistent with the GroEL experiments. 
Supplemental Figures and Tables.

Normal $(0.8 \mathrm{~mm})$ cone instrument pressures (mbar)

$\begin{array}{llllllll} & \text { Backing } & \text { Source } & \text { Trap } & \text { Helium Cell } & \text { IMS } & \text { Transfer } & \text { ToF } \\ \text { ToF mode } & 3.5 & 9.0 \mathrm{e}-3 & 3.6 \mathrm{e}-2 & 8.3 \mathrm{e}-4 & 1.0 \mathrm{e}-3 & 3.7 \mathrm{e}-2 & 1.5 \mathrm{e}-6 \\ \text { IMS mode } & 3.5 & 9.2 \mathrm{e}-3 & 4.2 \mathrm{e}-2 & 1.9 & 1.8 & 4.3 \mathrm{e}-3 & 1.7 \mathrm{e}-6\end{array}$

Medium $(0.67 \mathrm{~mm})$ cone instrument pressures (mbar)

$\begin{array}{llllllll} & \text { Backing } & \text { Source } & \text { Trap } & \text { Helium Cell } & \text { IMS } & \text { Transfer } & \text { ToF } \\ \text { ToF mode } & 2.4 & 6.2 \mathrm{e}-3 & 3.6 \mathrm{e}-2 & 8.3 \mathrm{e}-4 & 1.0 \mathrm{e}-3 & 3.7 \mathrm{e}-2 & 1.6 \mathrm{e}-6 \\ \text { IMS mode } & 2.4 & 6.4 \mathrm{e}-3 & 4.2 \mathrm{e}-2 & 1.9 & 1.8 & 4.3 \mathrm{e}-3 & 1.7 \mathrm{e}-6\end{array}$

Small $(0.45 \mathrm{~mm})$ cone instrument pressures (mbar)

$\begin{array}{llllllll} & \text { Backing } & \text { Source } & \text { Trap } & \text { Helium Cell } & \text { IMS } & \text { Transfer } & \text { ToF } \\ \text { ToF mode } & 1.4 & 3.6 \mathrm{e}-3 & 3.6 \mathrm{e}-2 & 8.2 \mathrm{e}-4 & 1.0 \mathrm{e}-3 & 3.7 \mathrm{e}-2 & 1.7 \mathrm{e}-6 \\ \text { IMS mode } & 1.4 & 3.8 \mathrm{e}-3 & 4.3 \mathrm{e}-2 & 1.9 & 1.8 & 4.4 \mathrm{e}-3 & 1.8 \mathrm{e}-6\end{array}$

Table S1. Instrument pressures for each source sampling cone in TOF mode and IMS mode. Each sampling cone provides a reproducible backing and source pressure reading, while all regions beyond the source are unchanged (from Trap to TOF). 


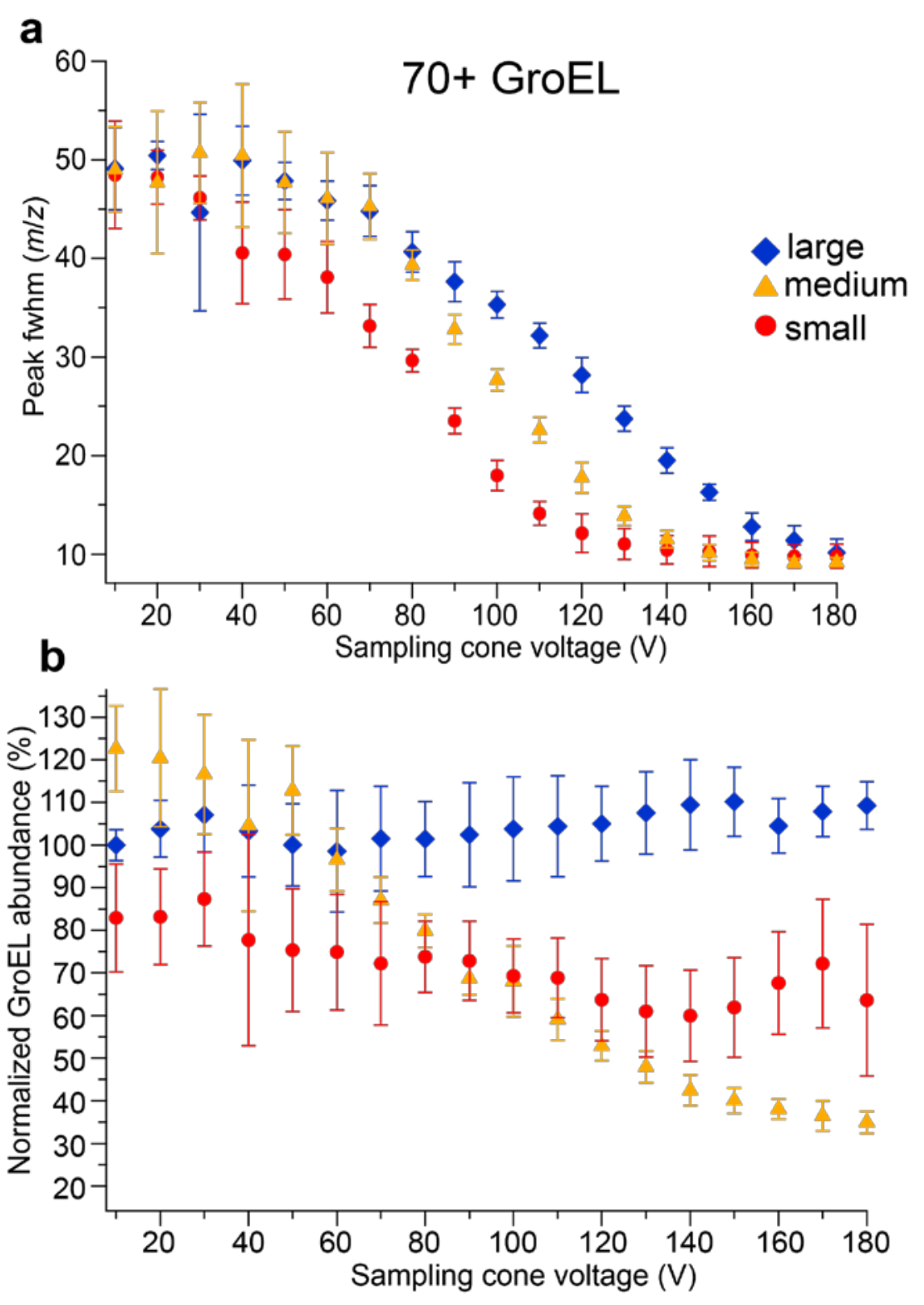

Figure S1. Peak width analysis for GroEL ${ }^{70+}$ ions (a) and total GroEL signal abundances with all charge states included (b). GroEL signal abundance for each point is normalized to that when using the large sampling cone at a cone potential of $10 \mathrm{~V}$. Note, with the medium and small cones, CID of GroEL 14-mers begins at $170 \mathrm{~V}$, which further reduces the signal. The error bars represent the standard deviation of the peak fwhm and abundance. 

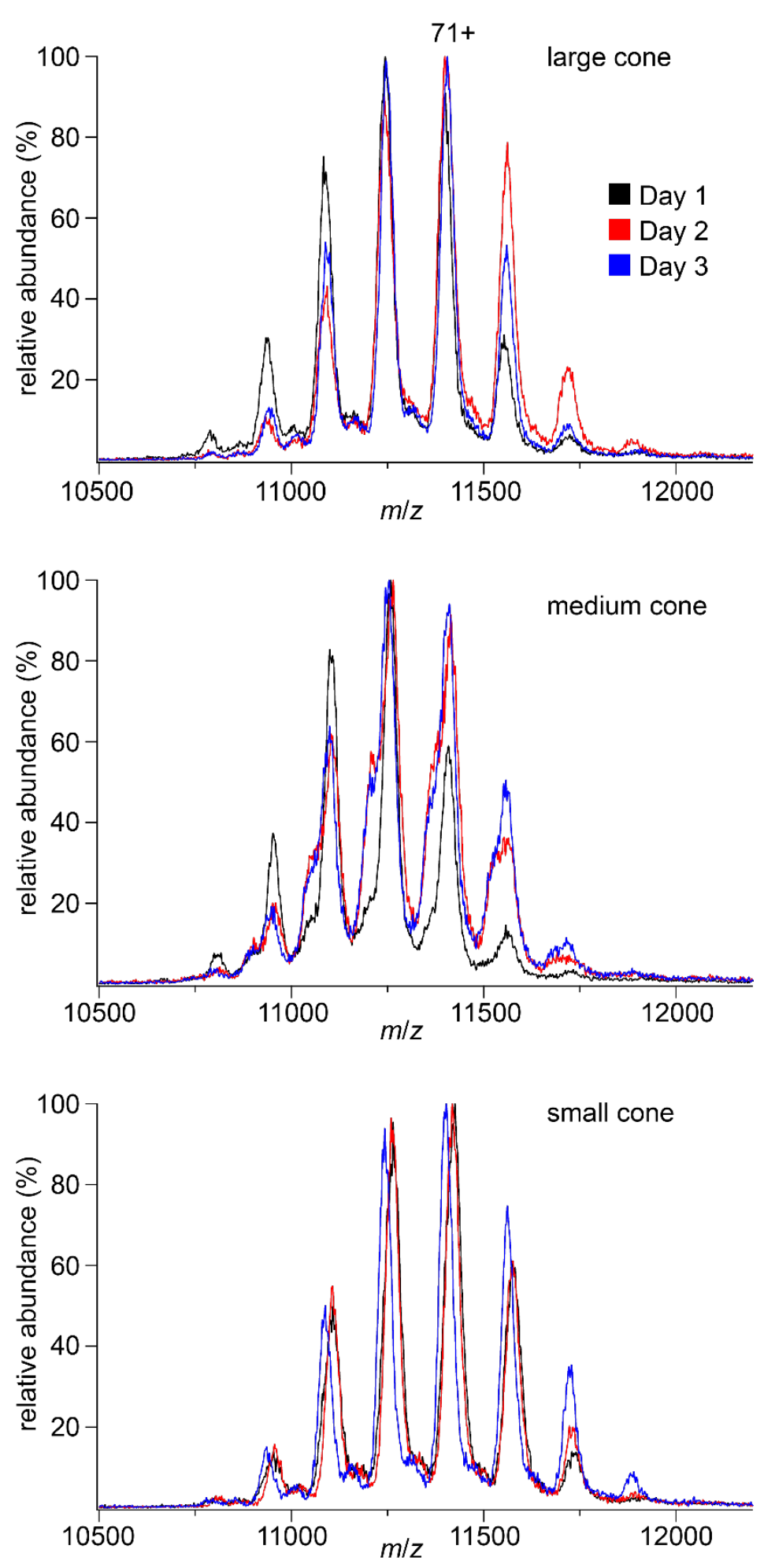

Figure S2. Overlaid mass spectra of GroEL for each cone and replicate at a low cone potential of $10 \mathrm{~V}$. 

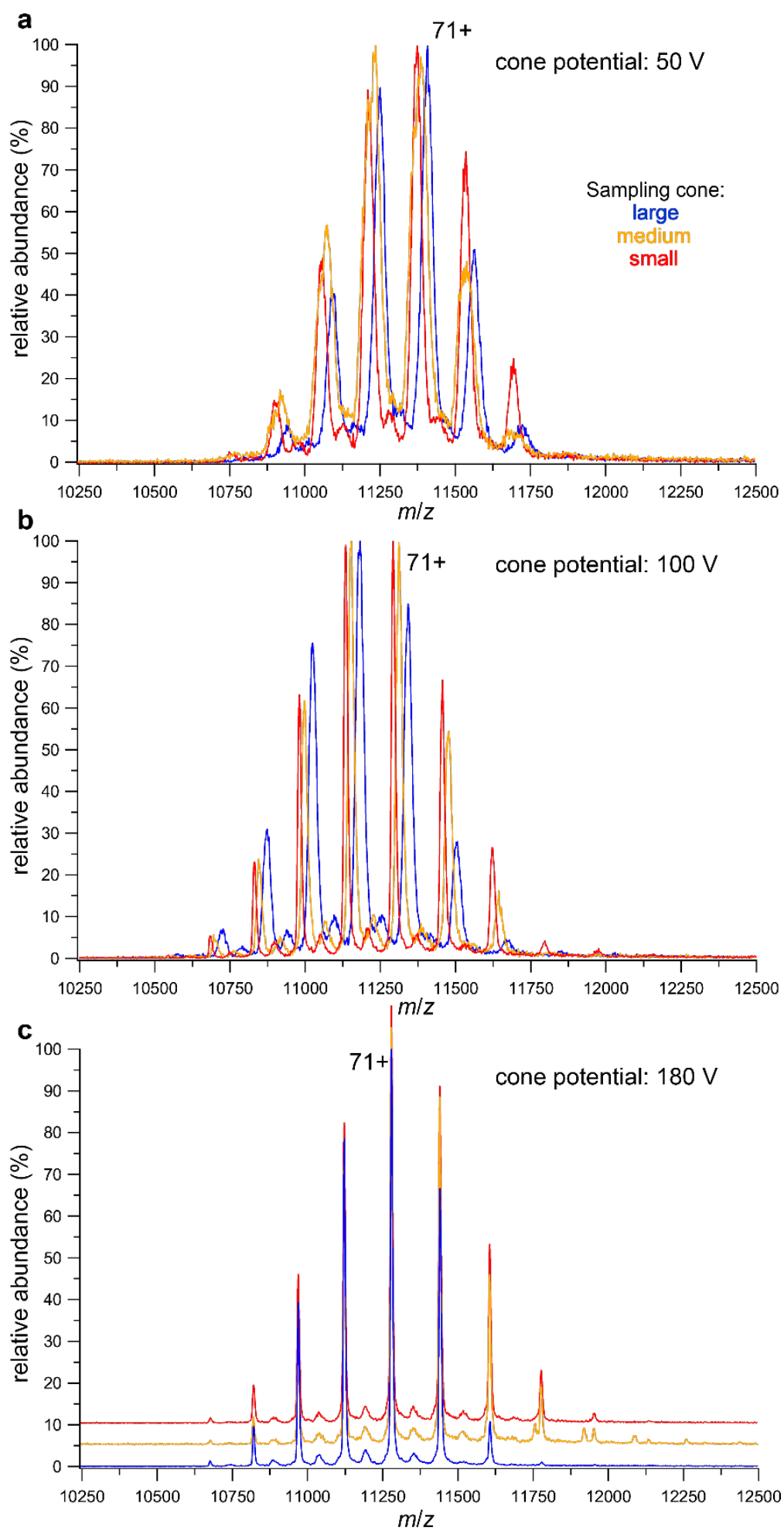

Figure S3. Selected mass spectra of GroEL 14-mers for each sampling cone overlaid at a sampling cone potential of $10 \mathrm{~V}(\mathrm{a}), 100 \mathrm{~V}$ (b), and $180 \mathrm{~V}$ (c). Note: in (c), mass spectra are slightly offset vertically to aid differentiation. 


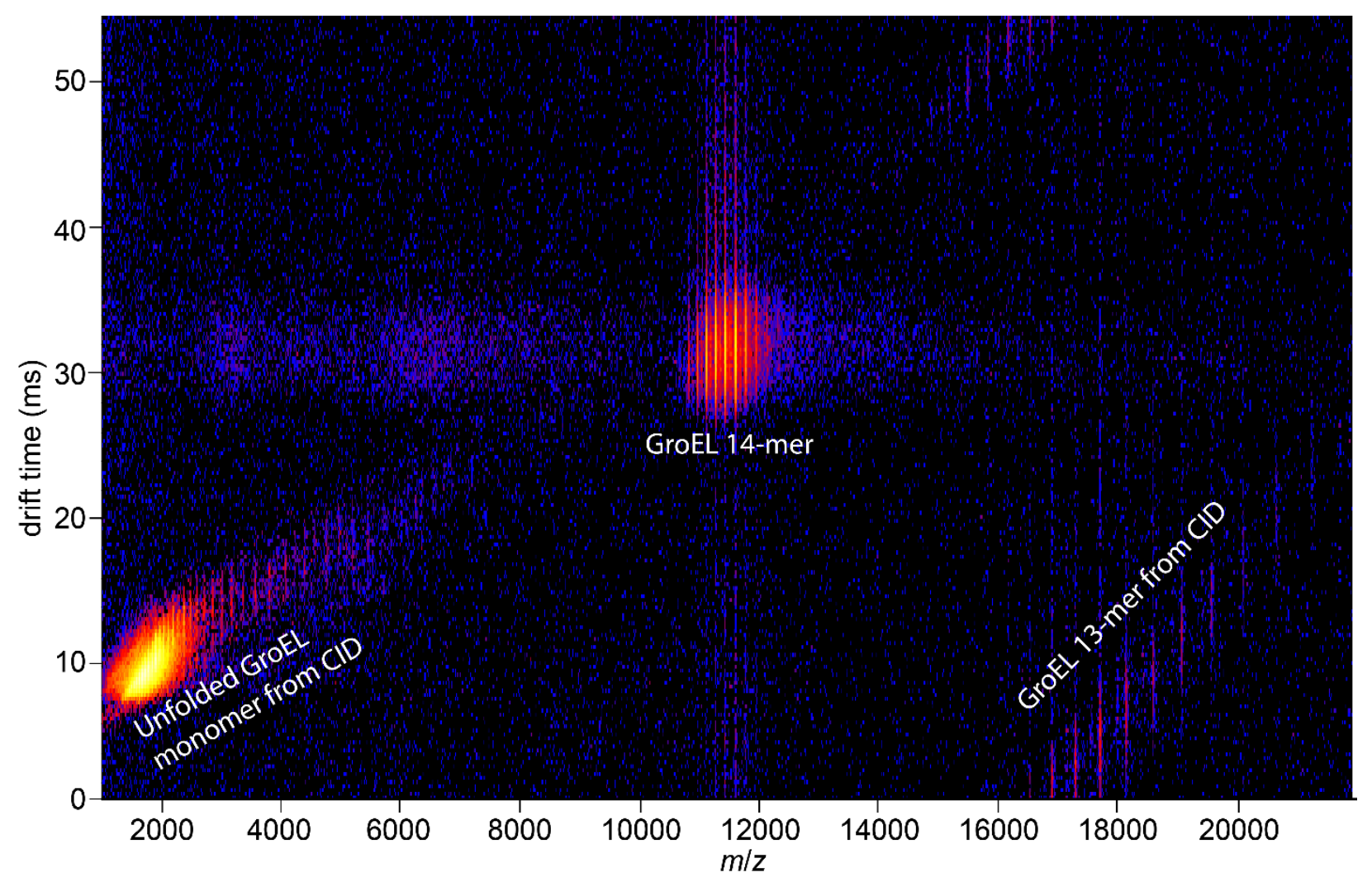

Figure S4. IM-MS spectrum of GroEL with the small cone at a cone potential of $200 \mathrm{~V}$. Under these conditions, activation in the source region is sufficient to cause CID of the GroEL 14-mer to produce high-charge monomer and GroEL 13-mer distributions. 

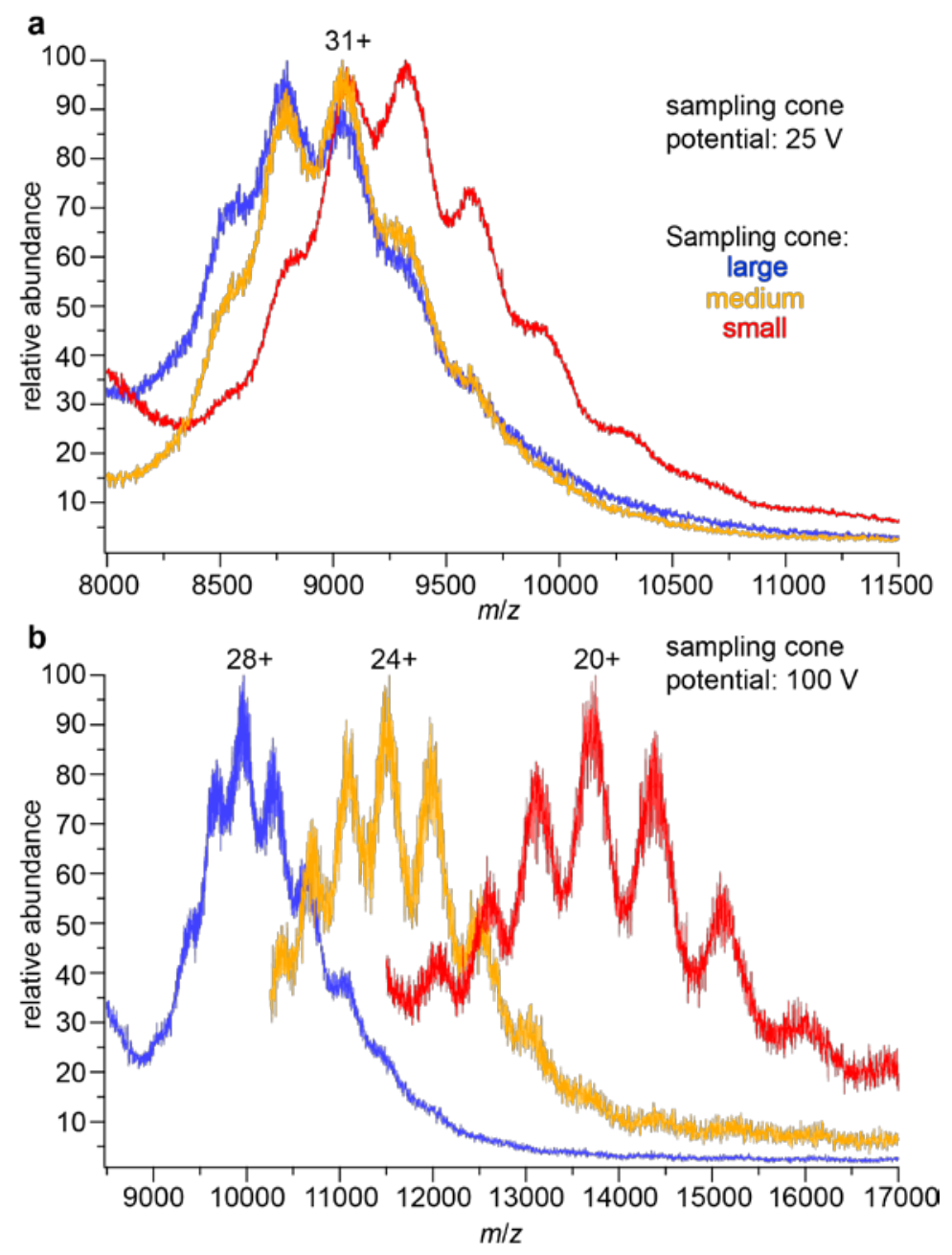

Figure S5. Overlaid mass spectra of $\alpha \mathrm{HL}$ complexes in FOS-14 micelles with each sample cone size at a sampling cone potential of $25 \mathrm{~V}$ (a) or $100 \mathrm{~V}(\mathrm{~b})$. Lower $\mathrm{m} / \mathrm{z}$ portions of the mass spectra collected with the medium and small cones are truncated for clarity due to increasing signal from empty FOS-14 micelles. The highest-abundance charge state for each spectrum is indicated. At low sampling cone potentials $(25 \mathrm{~V})$, the observed charge state and FOS-14 stoichiometry distributions show little dependence on sample cone diameter. As the potential is raised (100 V), the medium and small cones are more activating (strip more FOS-14 and charge) than the large cone. 

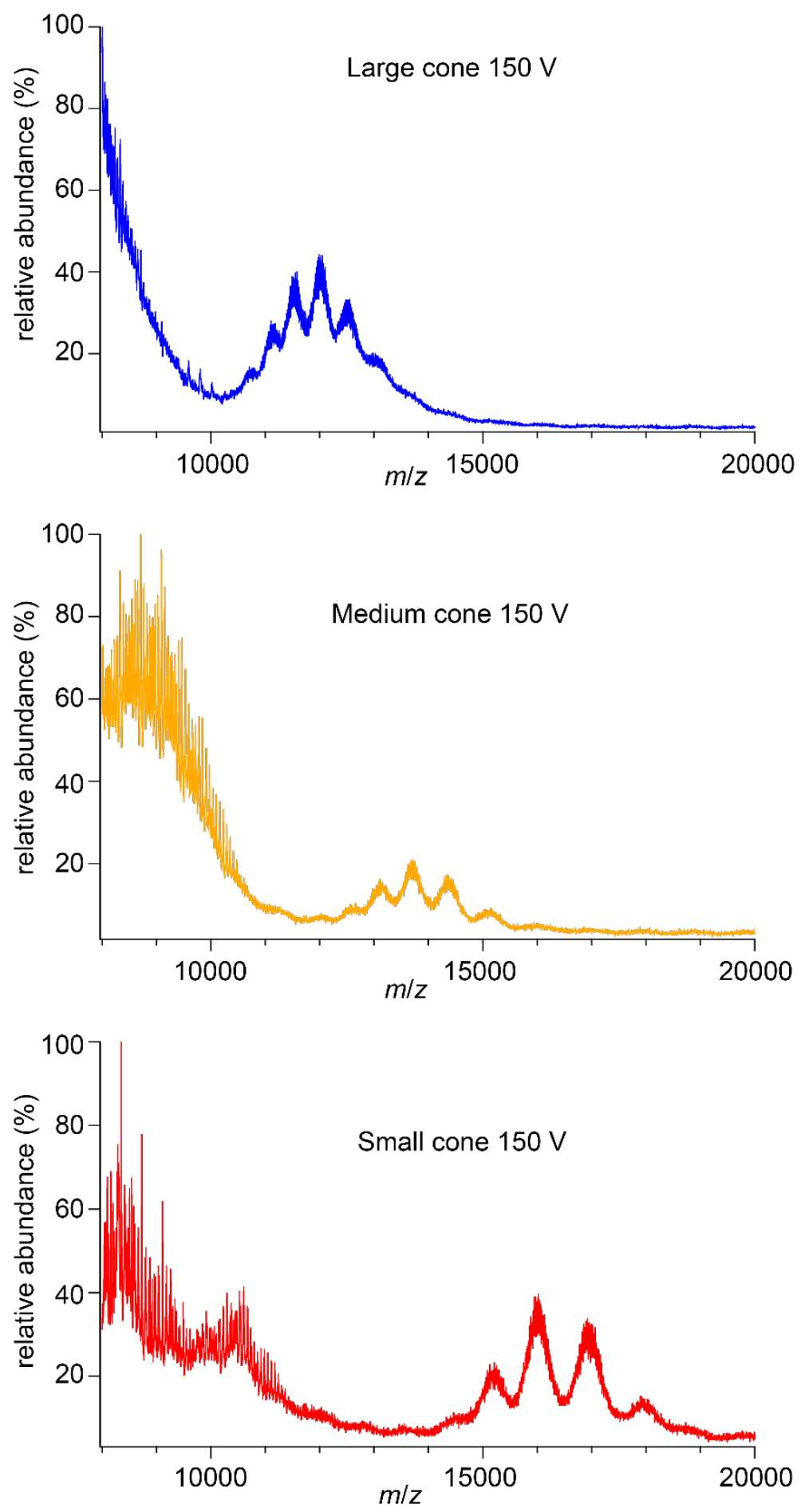

Figure S6. Extended $\mathrm{m} / \mathrm{z}$ range of $\alpha \mathrm{HL}$ FOS-14 complexes from Figure $2 \mathrm{a}$ (main text). The broad peak distribution below $\mathrm{m} / \mathrm{z} 10,000$ consists of primarily detergent clusters. 
a

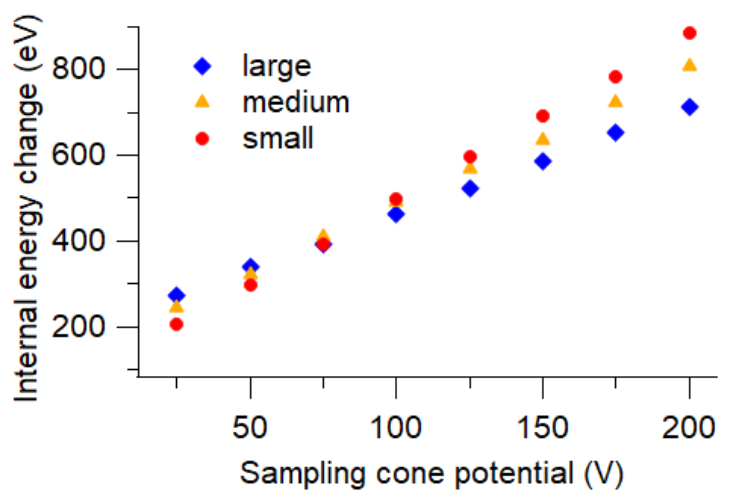

b

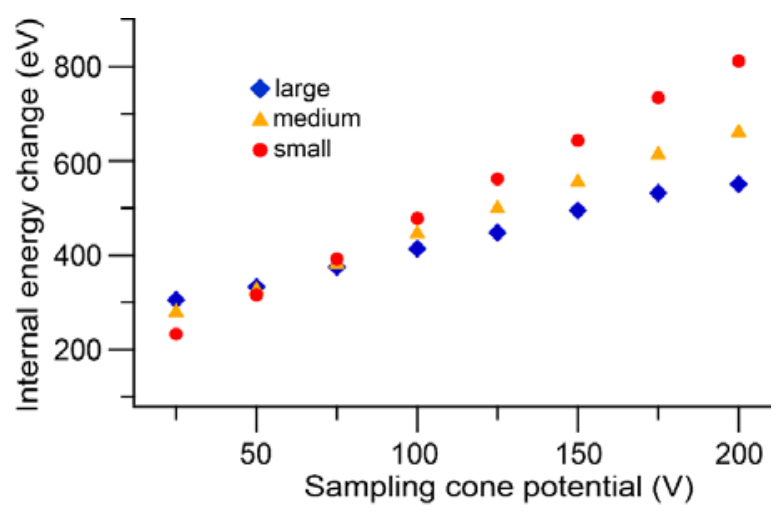

C

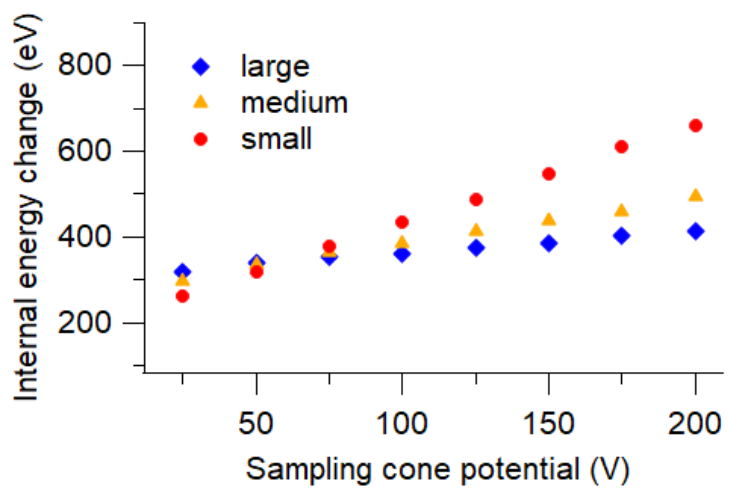

Figure S7. In the Stepwave, the sampling cone voltage setting accelerates ions through a differential aperture into a conjoined traveling-wave ion guide. With the large cone, the pressure before the aperture is -3.0 mbar and $\sim 9 \mathrm{e}-3 \mathrm{mbar}$ after the aperture, creating a high-pressure differential between the two regions. Above, simulated ion heating of $\mathrm{BSA}^{15+}$ under pressure conditions that match those produced by each sampling cone diameter as a function of acceleration potential, with short (a, $1.25 \mathrm{~mm})$, medium (b, 2.5 $\mathrm{mm}$ ), and long (c, $5.0 \mathrm{~mm}$ ) exponential pressure decay lengths as the ions are accelerated across the differential aperture. From these simulations of $\mathrm{BSA}^{15+}$ ions, little 
difference in effective ion internal temperature is predicted as a function of pressure at low cone potentials. However, the slopes of the ion heating trends increase as the pressure is reduced with the medium and small cones. For instance, at the medium exponential pressure decay length (b, $2.5 \mathrm{~mm}$ ), the slope is $1.5 \mathrm{x}$ and $2.25 \mathrm{x}$ the large cone for the medium and small cones, respectively. This means as the cone potential is raised at lower pressures, a higher percentage of collisions are net heating leading to a more efficient conversion of kinetic to internal energy, consistent with the GroEL

experiments. Decreasing the exponential decay length to $1.25 \mathrm{~mm}$ (a) brings the slopes of the ion heating trends closer together due to increased collisional cooling, while at a long decay length of $5.0 \mathrm{~mm}$ (c) the slopes diverge due to less collisional cooling. 


\begin{tabular}{cccccc} 
Cone potential & Cone size & $\begin{array}{c}\text { Avg. charge } \\
\text { state }\end{array}$ & Avg. mass (kDa) & $\begin{array}{c}\text { Bound FOS-14 } \\
\text { molecules }\end{array}$ & $\begin{array}{r}\text { fwhm FOS- } \\
\text { 14 molecules }\end{array}$ \\
& Large & $31.5 \pm 0.2$ & $280 \pm 1.2$ & $125 \pm 3$ & $20 \pm 4$ \\
& Medium & $30.9 \pm 0.3$ & $281 \pm 0.6$ & $126 \pm 2$ & $17 \pm 2$ \\
& Small & $30.6 \pm 0.3$ & $280 \pm 0.3$ & $126 \pm 1$ & $18 \pm 3$ \\
& & & & $122 \pm 4$ & $21 \pm 1$ \\
& Large & $27.9 \pm 0.3$ & $279 \pm 1.7$ & $118 \pm 2$ & $21 \pm 2$ \\
& Medium & $24.8 \pm 0.7$ & $277 \pm 0.9$ & $110 \pm 2$ & $21 \pm 1$ \\
& Small & $20.4 \pm 0.4$ & $275 \pm 0.5$ & & \\
& & & & & \\
& Large & $23.7 \pm 0.3$ & $276 \pm 0.9$ & $116 \pm 2$ & $22 \pm 1$ \\
& Medium & $20.3 \pm 0.4$ & $275 \pm 0.9$ & $111 \pm 2$ & $21 \pm 1$ \\
& Small & $17.0 \pm 0.3$ & $273 \pm 0.5$ & $105 \pm 1$ & $20 \pm 1$ \\
\hline
\end{tabular}

Table S2. GT analysis results for $\alpha \mathrm{HL}$ heptamers in FOS-14 micelles taken in triplicate with each sampling cone. The \pm corresponds to the standard deviation of the triplicate measurement. 